\title{
Salivary Atopy Biomarkers in Patients with Geographic Tongue
}

\author{
Leila Farhad-Mollashahi ${ }^{1}$ Zohreh Dalirsani ${ }^{10} \quad$ Marieh Honarmand $^{2}$ Saeedeh Salimi ${ }^{3}$ \\ Soudeh Shahabi Nezhad ${ }^{4}$
}

1 Oral and Maxillofacial Diseases Research Center, Mashhad University of Medical Sciences, Mashhad, Iran

2 Oral and Dental Disease Research Center, Department of Oral Medicine, School of Dentistry, Zahedan University of Medical Sciences, Zahedan, Iran

${ }^{3}$ Department of Biochemistry, School of Medicine, Zahedan University of Medical Sciences, Zahedan, Iran

${ }^{4}$ General Dentist, Zahedan, Iran
Address for correspondence Marieh Honarmand, DDS, MSc, Oral and Dental Disease Research Center, Department of Oral Medicine, School of Dentistry, Zahedan University of Medical Sciences, Zahedan, Iran (e-mail: honarmandmarieh56@gmail.com).

\begin{abstract}
Keywords

- Geographic tongue

- immunoglobulin E

- eosinophil cationic protein

- saliva

- atopy
\end{abstract}

Objectives Geographic tongue is the mucositis of the dorsal surface of the tongue with no definite etiology, although it may be associated with atopic conditions. In this study, the salivary immunoglobulin $\mathrm{E}(\mathrm{IgE})$ and eosinophilic cationic protein (ECP) levels were assessed in patients with geographic tongue to examine the relationship between this condition and allergy-related biomarkers.

Materials and Methods In this case-control study, unstimulated saliva was collected from 45 geographic tongue patients and 45 controls, and the salivary IgE and ECP levels were assessed. The data were analyzed with SPSS 20.0 using the Mann-Whitney test with a significance level of $p<0.05$.

Results The salivary IgE level in the case and control groups was $123.76 \pm 8.1$ and $74.34 \pm 6.2 \mathrm{IU} / \mathrm{mL}$, respectively, and the salivary ECP level was $9.4 \pm 6.0$ and $7.6 \pm 3.2$ $\mathrm{ng} / \mathrm{mL}$, respectively. There was a statistically significant difference between the two groups in terms of salivary $\operatorname{lgE}(p=0.007)$ and ECP $(p=0.001)$ levels.

Conclusion Salivary IgE and ECP levels increase in patients with geographic tongue. They can, therefore, be used for the initial diagnostic workup and to investigate the possible association of geographic tongue with allergic reactions.

\section{Introduction}

Geographic tongue is characterized by round and irregular depapillated zones on the tongue with distinctive whitish hyperkeratotic borders. ${ }^{1,2}$

Most patients are asymptomatic but some may complain of pain and burning sensation. The etiology of geographic tongue is unknown. However, according to some reports, it is associated with gastrointestinal diseases, candidiasis, atopy, anxiety, ${ }^{3,4}$ and psoriasis. ${ }^{5}$

Previous studies have examined the levels of biomarkers associated with allergic reactions in atopic patients. In Rasheed et al, the serum levels of some biomarkers, including the filaggrin protein, eosinophil major basic protein (MBP)
DOI https://doi.org/ $10.1055 / \mathrm{s}-0041-1736375$. ISSN 2278-9626.

\footnotetext{
(c) 2021. European Journal of General Dentistry. All rights reserved. This is an open access article published by Thieme under the terms of the Creative Commons Attribution-NonDerivative-NonCommercial-License, permitting copying and reproduction so long as the original work is given appropriate credit. Contents may not be used for commercial purposes, or adapted, remixed, transformed or built upon. (https://creativecommons.org/ licenses/by-nc-nd/4.0/)

Thieme Medical and Scientific Publishers Pvt. Ltd., A-12, 2nd Floor, Sector 2, Noida-201301 UP, India
} 
and total immunoglobulin E (IgE), have been reported to be higher in patients with atopic conditions such as atopic dermatitis (AD), allergic rhinitis (AR) and bronchial asthma (BA). In addition, the level of biomarkers was also higher in patients with higher disease scores than in patients with milder diseases. ${ }^{6}$ This indicates the possible association of these biomarkers with the development and severity of allergic conditions.

Another biomarker that has been evaluated in some allergic conditions is the eosinophil cationic protein (ECP), a nonspecific marker released by eosinophils in allergic reactions, which is found in plasma, saliva, urine, nasal secretions, and other body fluids. ${ }^{7}$

The biological activities of ECP include the suppression of the proliferative responses of $\mathrm{T}$ cells, the synthesis of immunoglobulin by B cells, the degranulation of mast cells, the regulation of fibroblast activity, the stimulation of airway mucus secretion, and the disruption of the coagulation and complement system. However, the most important function of ECP is cytotoxic activity against bacteria, fungi, viruses, and respiratory epithelial and cancer cells. The cytotoxic activity of ECP is exerted by the formation of pores in the cell membrane to penetrate water and small molecules into the cell and lead to the osmotic lysis of the target cell. ${ }^{8,9}$

ECP plays an important role in the pathogenesis of some atopic conditions such as allergic rhinitis, ${ }^{7}$ and its level may increase in allergic conditions such as recurrent wheezing ${ }^{10}$ and rhinosinusitis. ${ }^{11}$

Kirgezen et al found in their 2019 study on patients with allergic rhinitis that the IgE D2 levels were higher in patients than in controls, and that the salivary and serum ECP levels were not significantly different. ${ }^{7}$ Therefore, by examining the salivary level of ECP, the serum level can be predicted approximately. In contrast, Amin et al showed that not only was the serum level of ECP higher in patients with allergic rhinitis but there was also a significant relationship between the serum level of this biomarker and that of IgE. ${ }^{12}$

According to the study by Rezaei et al, the salivary IgE was significantly higher in patients with geographic tongue than in controls, although there was no significant difference in the total antioxidant capacity (TAC) and catalase activity between the two groups. This indicates a stronger association of the condition with the atopic status and lesser association with the antioxidant capacity. ${ }^{13}$

It is important to identify effective factors in allergic conditions when using the results of these studies in treatment. In recent years, some researchers have proposed immunotherapy for allergic conditions. Djuric-Filipovic et al found that immunotherapy was effective not only on symptoms of asthma and allergic rhinitis in children but also improved lung function and reduced airway inflammation. ${ }^{14}$

Given the possible link between allergic reactions and the development of geographic tongue as well as the lack of studies evaluating allergy-related biomarkers, the present study was designed to investigate two factors that imply allergic conditions in people with geographic tongue as an atopic condition. If an association could be established between the development of geographic tongue with an increase in these factors, it would advocate the hypothesis of the development of geographic tongue after allergic conditions. It can therefore be useful for prevention or treatment process.

\section{Methods and Materials}

\section{Study Subjects}

In this case-control study, 45 patients with geographic tongue and 45 healthy individuals referred to the Faculty of Dentistry at Zahedan University of Medical Science in Iran were selected, according to consecutive sampling. The controls matched the case group in terms of age and gender.

Inclusion criteria were written informed consent and geographic tongue confirmed by diseases history and clinical examination. Exclusion criteria were any systemic disease, either allergic or atopic, such as asthma, allergic rhinitis, atopic dermatitis, eczema, parasitic infections, etc., use of medication in the last three months, pregnancy, tobacco and alcohol consumption, and other diseases of the oral mucosa.

The oral mucosa was examined using a disposable mirror under the light of the dental unit and in accordance with the World Health Organization (WHO) guidelines. Geographic tongue was identified, based on the loss of local filiform papillae with irregular borders, migrate over time. ${ }^{15}$

\section{Saliva Collection}

Unstimulated saliva was collected by spitting method. Participants were asked not to eat, drink, or brush their teeth 90 minutes before collecting saliva. They bent their heads forward and spitted saliva into the test tube for 10 to 15 minutes in a sterile $5 \mathrm{~mL}$ centrifuge tube. ${ }^{16}$ Samples were collected between 9 to 11 a.m. After collecting saliva samples, the test tubes were tightly closed with paraffin and labeled accordingly. The samples were immediately transported to the laboratory, where they were centrifuged at $2000 \mathrm{rpm}$ for 10 minutes to separate the debris. The samples were then transferred to microtubes, labeled and stored at $-80{ }^{\circ} \mathrm{C}$ for the experiment. The salivary IgE and ECP levels were evaluated according to the ELISA method using available kits (Radim S.p.a, Italy and Chongqing Biospes Co., China).

All participants signed an informed consent. The study design was approved by the Ethical Committee of Zaheden University of Medical Sciences under the code IR.ZAUMS. REC.1393.1084.

\section{Statistical Analysis}

The data was analyzed using the SPSS 20.0 software. Due to the non-normality of the data, the Mann-Whitney test was used to compare salivary ECP and IgE levels in the two groups. The significance level was set at $p<0.05$.

\section{Results}

A total of 90 people participated in this study, 45 with geographic tongue (22 men and 23 women) with a mean age of 32 years as the case group and 45 healthy individuals 
Table 1 Demographic characteristics and IgE and ECP levels of case and control groups

\begin{tabular}{|l|l|l|l|}
\hline Variable & Case group & Control group & $p$-Value \\
\hline $\begin{array}{l}\text { Gender } \\
\text { (female/male) }\end{array}$ & $23 / 22$ & $18 / 27$ & $0.230^{\mathrm{a}}$ \\
\hline Age (years) & 32 & 31 & $0.264^{\mathrm{b}}$ \\
\hline $\mathrm{IgE} \mathrm{level} \mathrm{IU/mL}$ & $123.76 \pm 8.1$ & $74.34 \pm 6.2$ & $0.007^{\mathrm{c}}$ \\
\hline ECP level $\mathrm{ng} / \mathrm{mL}$ & $9.4 \pm 6$ & $7.6 \pm 3.2$ & $0.001^{\mathrm{c}}$ \\
\hline
\end{tabular}

Abbreviations: ECP, eosinophilic cationic protein; IgE, immunoglobulin E. ${ }^{\mathrm{a}}$ Chi-square

bIndependent $t$-test

${ }^{c}$ Mann-Whitney test

(27 men and 18 women) with a mean age of 31 years as controls. The two groups matched in terms of age and gender (-Table 1). The ECP and IgE levels in the case group were higher than those in controls. According to the MannWhitney test, there was a statistically significant relationship between geographic tongue involvement and increased salivary ECP and IgE levels $(p=0.001$ and $p=0.007$, respectively) (-Table $\mathbf{1}$ ).

\section{Discussion}

Geographic tongue is a common inflammatory condition that affects the dorsal surface and lateral borders of the tongue. ${ }^{13}$ Although the etiology of geographic language is unknown, allergy is considered to be the most important etiological factor. ${ }^{17,18}$ So far there have been several studies examining the relationship between geographic tongue and allergies. They have mostly focused on either prick and patch tests ${ }^{19}$ or questionnaires and self-reports, ${ }^{20}$ while less attention was paid to allergy-related biomarkers. For example, the study by Goregen et al examined allergies in patients with geographic tongue using patch and prick tests. Based on the results, the positivity rate of the two tests was $47.5 \%$ in the case group and $22.5 \%$ in controls, which showed a significant increase in the susceptibility to allergies in the case group compared with the controls. ${ }^{19}$ However, in the study by Shulman et al, who investigated the prevalence of risk factors related to geographic tongue in American adults, there was no significant relationship between allergic factors and geographic tongue. ${ }^{15}$

Given the possibility of an association between allergies and geographic tongue suggested in previous studies, we examined in this study the salivary IgE and ECP as two known biomarkers for allergies. ECP is a mediator of allergic inflammation, which is released by eosinophils and is activated by IgE. ${ }^{21}$ Eosinophils contain granules with chemical mediators such as MBP-1, MBP-2, ECP, and eosinophil-derived neurotoxin (EDN). These mediators cause tissue damage and stimulate basophils, mast cells, and neutrophils. Activated eosinophils induces several cytokines and chemokines such as interleukin (IL)-16, IL-12, IL-13, and TGFB1, which play an important role in regulating immunity. ${ }^{22}$
ECP is found in various body fluids such as serum, plasma, sputum, saliva, nasal secretions and urine, and its level increases in atopic conditions such as allergic rhinitis, recurrent wheezing as well as in chronic infections such as chronic rhinosinusitis. $^{7}$

The association between ECP levels and oral lesions has been less studied. One study showed that salivary ECP levels in patients with recurrent aphthous stomatitis were significantly higher than in healthy individuals. ${ }^{23}$ In the study by De Lima et al, the effects of ECP on oral squamous cell carcinoma (OSCC) cell lines were assessed and a significant inverse relationship between ECP concentrations and the viability of SCC- 4 and SCC-25 cells was observed. ${ }^{8}$

Various studies have shown an association between ECP and allergic conditions. ${ }^{7,24-26}$ A study on atopic dermatitis showed that the level of serum ECP was significantly higher during the acute phase of the disease compared with the controls, also it decreased along with the improvement of symptoms after treatment. ${ }^{24} \mathrm{~A}$ study on children with atopic dermatitis also showed a decrease in serum ECP levels and an improvement in the clinical symptoms following a reduction in the consumption of processed foods. ${ }^{25}$

Schmekel et al found that salivary ECP levels were higher in asthmatic patients and decreased with increasing dose of inhaled corticosteroids. This indicates the presence of eosinophils in the oral mucosa and salivary glands of asthmatic patients and increased permeability of the oral mucosa due to the destructive effects of eosinophils or increased peripheral serum ECP levels. $^{26}$

Our study showed that ECP is significantly associated with geographic tongue. However, there is no evidence that can explicitly confirm or rule out this association, as only the association of ECP with other allergic conditions such as asthma, allergic rhinitis, atopic dermatitis and food allergies have been studied so far.

IgE is a molecule, which activates cells involved in allergic inflammation such as eosinophils and basophils; therefore it plays a role in the pathogenesis of atopic conditions. ${ }^{22}$ In the study performed by Kirgezen et al, serum specific IgE D2 in patients with allergic rhinitis was significantly higher than in the controls. In addition, the salivary and serum ECP levels were higher in patients, although there was no significant difference. ${ }^{7}$ Rezaei et al reported that the salivary IgE level in patients with geographic tongue was significantly higher than that of controls, ${ }^{13}$ which is consistent with the results of the present study.

Assessing salivary biomarkers is an easy, fast, inexpensive, noninvasive, painless and minimally-risky diagnostic approach, which can play a role in diagnosing systemic and oral diseases. ${ }^{27,28}$ Saliva provides significant information about general and oral health. Therefore, examining salivary biomarkers associated with diseases such as atopy can help assess the severity of the disease and identify possible causes of symptoms. ${ }^{29}$

Briefly, and according to the results of this study, salivary IgE and ECP levels increase in patients with geographic tongue. These biomarkers can therefore be used for the initial workup in patients with this condition. 


\section{Conflict of Interest}

The authors declare no conflict of interest.

\section{Acknowledgments}

The authors would like to extend their appreciation to the vice chancellor for research, and to Zahedan University of Medical Sciences for the financial support. The results described in this paper was part of a (D.D.S.) student thesis.

\section{References}

1 Ahmad P, Akhtar U, Chaudhry A, et al. Repercussions of diabetes mellitus on the oral cavity. Eur J Gen Dent 2019;8(03):55

2 Khan S, Zaheer S, Gupta N. Oral psoriasis: a diagnostic dilemma. Eur J Gen Dent 2013;2(01):67

3 González-Álvarez L, García-Pola MJ, Garcia-Martin JM. Geographic tongue: Predisposing factors, diagnosis and treatment. A systematic review. Rev Clin Esp (Barc) 2018;218(09):481-488 (English Edition)

4 Honarmand M, Farhad Mollashahi L, Shirzaiy M, Sehhatpour M. Geographic tongue and associated risk factors among Iranian dental patients. Iran J Public Health 2013;42(02):215-219

5 Barros FC, Sampaio JN, Figueredo CMDS, Carneiro S, Fischer RG. Higher prevalence of periodontitis and decayed, missing and filled teeth in patients with psoriasis. Eur J Dent 2020;14(03): 366-370

6 Rasheed Z, Zedan K, Saif GB, et al. Markers of atopic dermatitis, allergic rhinitis and bronchial asthma in pediatric patients: correlation with filaggrin, eosinophil major basic protein and immunoglobulin E. Clin Mol Allergy 2018;16:23

7 Kırgezen T, Server EA, Turanoğlu FS, Yiğit Ö, Uzun H, Durmuş S Salivary eosinophil cationic protein in allergic rhinitis. Turk Arch Otorhinolaryngol 2019;57(02):91-94

8 DE Lima PO, Dos Santos FV, Oliveira DT, DE Figueiredo RC, Pereira MC. Effect of eosinophil cationic protein on human oral squamous carcinoma cell viability. Mol Clin Oncol 2015;3(02):353-356

9 Pereira MC, Oliveira DT, Olivieri EH, et al. The $434(\mathrm{G}>\mathrm{C})$ polymorphism in the eosinophil cationic protein gene and its association with tissue eosinophilia in oral squamous cell carcinomas. J Oral Pathol Med 2010;39(01):56-62

10 Yu J, Yoo Y, Kim DK, Kang H, Koh YY. Bronchial responsiveness and serum eosinophil cationic protein levels in preschool children with recurrent wheezing. Ann Allergy Asthma Immunol 2005;94 (06):686-692

11 Kim KS, Won H-R, Park CY, et al. Analyzing serum eosinophil cationic protein in the clinical assessment of chronic rhinosinusitis. Am J Rhinol Allergy 2013;27(03):e75-e80

12 Amin K, Issa SM, Ali KM, et al. Evidence for eosinophil and IL-17 mediated inflammation in allergic rhinitis. Clin Mol Allergy 2020; 18:6

13 Rezaei F, Fatholahi S, Rezaei F. Assessment of salivary antioxidant status and immunoglobulin $\mathrm{E}$ in patients with geographic tongue. J Family Med Prim Care 2020;9(01):72-76
14 Djuric-Filipovic I, Caminati M, Filipovic D, Salvottini C, Zivkovic Z. Effects of specific allergen immunotherapy on biological markers and clinical parameters in asthmatic children: a controlled-real life study. Clin Mol Allergy 2017;15:7

15 Shulman JD, Carpenter WM. Prevalence and risk factors associated with geographic tongue among US adults. Oral Dis 2006;12 (04):381-386

16 Shirzaiy M, Aiub Rigi Ladiz M, Dalirsani Z, Dehghan Haghighi J Nakhaii A. Evaluation of salivary total antioxidant capacity in smokers with severe chronic periodontitis. Int J High Risk Behav Addict 2017;6(03):e59486

17 Minciullo PL, Paolino G, Vacca M, Gangemi S, Nettis E. Unmet diagnostic needs in contact oral mucosal allergies. Clin Mol Allergy 2016;14(01):10

18 Darling MR, Su N, Masen S, et al. Geographic tongue: assessment of peripheral nerve status, Langerhans cell, and HLA-DR expression. Oral Surg Oral Med Oral Pathol Oral Radiol 2017;124(04): 371-377.e1

19 Goregen M, Melikoglu M, Miloglu O, Erdem T. Predisposition of allergy in patients with benign migratory glossitis. Oral Surg Oral Med Oral Pathol Oral Radiol Endod 2010;110(04):470-474

20 Jainkittivong A, Langlais RP. Geographic tongue: clinical characteristics of 188 cases. J Contemp Dent Pract 2005;6(01):123-135

21 Keleş E, Yazgan H, Gebeşçe A. To evaluate serum eosinophil cationic protein and total ige concomitantly may predict the persistence of wheezing in young children. ISRN Pediatr 2012; 2012:168379

22 Hu Y, Liu S, Liu P, Mu Z, Zhang J. Clinical relevance of eosinophils, basophils, serum total IgE level, allergen-specific IgE, and clinical features in atopic dermatitis. J Clin Lab Anal 2020;34(06):e23214

23 Farhad-Mollashahi L, Honarmand M, Nakhaee A, Kamalzadeh S, Amini S. Salivary levels of IgE and ECP in patients with recurrent aphthous stomatitis. J Clin Exp Dent 2020;12(01):e9-e12

24 Angelova-Fischer I, Hipler UC, Bauer A, et al. Significance of interleukin-16, macrophage-derived chemokine, eosinophil cationic protein and soluble E-selectin in reflecting disease activity of atopic dermatitis-from laboratory parameters to clinical scores. Br J Dermatol 2006;154(06):1112-1117

25 Lee JM, Jin HJ, Noh G, Lee SS. Effect of processed foods on serum levels of eosinophil cationic protein among children with atopic dermatitis. Nutr Res Pract 2011;5(03):224-229

26 Schmekel B, Ahlner J, Malmström M, Venge P. Eosinophil cationic protein (ECP) in saliva: a new marker of disease activity in bronchial asthma. Respir Med 2001;95(08):670-675

27 Rabiei M, Asli HN, Mohamadi MH. Comparison of salivary calcium level in dentulous and edentulous patients. Eur J Dent 2019;13 (01):36-41

28 Khurshid Z, Moin SF, Khan RS, Agwan MAS, Alwadaani AH Zafar MS. Human salivary protein extraction from RNAPro.SAL, Pure.SAL, and passive drooling method. Eur J Dent 2017;11(03): 385-389

29 Roi A, Rusu LC, Roi CI, Luca RE, Boia S, Munteanu RI. A new approach for the diagnosis of systemic and oral diseases based on salivary biomolecules. Dis Markers 2019;2019:8761860 